\title{
On the Charges and Currents in the Quantum Field Theory
}

\author{
Daniel Sepunaru \\ RCQCE-Research Center for Quantum Communication, Holon Academic Institute of Technology, Holon, Israel \\ Email: danielsepunaru@walla.co.il
}

Received 7 January 2016; accepted 9 June 2016; published 14 June 2016

Copyright (C) 2016 by author and Scientific Research Publishing Inc. This work is licensed under the Creative Commons Attribution International License (CC BY). http://creativecommons.org/licenses/by/4.0/

cC) (i) Open Access

\section{Abstract}

This paper is concerned with the determination of currents and charges in hypercomplex extensions of the Feynman-Dyson derivation of the Maxwell-Faraday equations. We analyze the appearance of charges and currents in non-Abelian versions of that approach: SU(2), SU(3) and G2. The structure constants of G2 Lie algebra are computed explicitly. Finally, we suggest a seven-dimensional treatment of color.

\section{Keywords}

Gauge Charges, Structure Constants, Multiplication Tables, Color

\section{Introduction}

This paper is a continuation of the discussion on hypercomplex extensions of the Feynman-Dyson derivation of the Maxwell-Faraday equations. Usually mathematical proofs have only relatively minor value since for any set of mathematical arguments it is possible to present an equally valuable set of contra-arguments; in physics, by contrast, the ultimate verification of a statement is its confirmation by experiment and the solution is unique.

The specific topic of the present discussion is the determination of currents and charges in the suggested schemas [1]-[4]. As the defining model we consider the structure of the classical electrodynamics which consists of two parts: the first, the "inertial" fields produced by a moving source that is wrapped around the source, and the second, the radiated part that consist of the excessive field due to the accelerated motion of the source. Equivalently, the solutions may be viewed as a description of the motion of a source under the influence of an external field. The role of the inhomogeneous equations in all that is crucial. They define the electromagnetic parameters of the source: charge and current. This is not "merely" a definition, for it produces a drastic change in the physical content of the theory which leads to reconsideration of the structure of the space-time continuum (a change from Galilean to Lorentz group transformations that leave the equations invariant). It also introduces a 
new type of symmetry-internal local gauge symmetry.

Thus, charge and the current turn out to be newly conserved quantities. Note that the non-Abelian extension of the gauge fields proceeds through the steps described above [3]. Therefore a more detailed analysis of what has been done so far is required as well as an explanation of the reasoning behind it.

\section{Mathematical Preliminary.}

Mathematical background for our discussion is necessary.

Since we are interested in a theory with uniquely determined predictions, it is advisable to use a numeric system that allows that to occur: normed division algebras which include the real (1dimensional), complex (2 dimensional), quaternion (4 dimensional) and octonion (8 dimensional) algebras that satisfy the definition of quadratic composition algebras:

$$
\begin{gathered}
x^{2}-\operatorname{Tr}(x) x+N(x) 1=0, \forall x=\text { real, complex, quaternion, octonion } \\
x+\bar{x} \equiv \operatorname{Tr}(x) 1, \operatorname{Tr}(x)=\text { real, } \\
x \bar{x}(=\bar{x} x) \equiv N(x) 1, \quad N(x)=\text { real. }
\end{gathered}
$$

Definitions of trace $\operatorname{Tr}(x)$ and norm $N(x)$ are consistent with those of matrix calculus.

Note that in contrast with reals, complex and quaternions, octonions are non-associative (but still alternative) algebra and therefore can't be represented by matrices.

Now we need to introduce multidimensional numeric objects-vectors to extend the usual arithmetic operations-addition, multiplication by a constant number, and multiplication between them. In so doing, we now gain three types of multiplication:

1) scalar multiplication described by the Jordan product

$$
\boldsymbol{A} \cdot \boldsymbol{B} \equiv \frac{1}{2}(\boldsymbol{A B}+\boldsymbol{B} \boldsymbol{A})
$$

which maps vector fields into scalars;

2) vector multiplication described by the Lie bracket product

$$
\boldsymbol{A} \times \boldsymbol{B} \equiv \frac{1}{2}(\boldsymbol{A B}-\boldsymbol{B} \boldsymbol{A})
$$

which maps vector fields into vectors; and

3) tensor multiplication $\boldsymbol{A} \otimes \boldsymbol{B}$ which leads to the higher dimensional algebras.

Surprisingly, vector multiplication does not always satisfy the usually required properties [5] [6].

It realized consistently only in $n=1, n=3$ and $n=7$ dimensional space according to [7] [8]:

$$
n(n-1)(n-3)(n-7)=0,
$$

where $n$ is the dimension of the underlined vector space.

Bearing in the mind the definition of charge in the following discussion, from now on, by the term multiplication we mean the Lie bracket product

$$
e_{a} \times e_{b}=\frac{1}{2}\left(e_{a} e_{b}-e_{b} e_{a}\right) .
$$

For the quaternions $(n=3)$ the structure constants $f_{a b c}$ may be computed from

$$
e_{a} \times e_{b} \equiv \frac{1}{2}\left[e_{a}, e_{b}\right]=f_{a b c} e_{c} ; \quad a, b, c=1,2,3
$$

where $f_{a b c}=\varepsilon_{a b c}$, and $\varepsilon_{a b c}$ is totally antisymmetric Levi-Civita symbol with the only nonzero independent components $\varepsilon_{123}=1$. Thus we get quaternion multiplication table (see Figure 1), where $e_{a}^{2}=-1 ; a=1,2,3$. From (1) it follows that $e_{a}$ are the traceless, antihermition generators of quaternion algebra. We compute the quaternion and octonion multiplication tables in order to compare them with the corresponding commutation 


$$
\begin{array}{cccc}
e_{a} / e_{b} & e_{1} & e_{2} & e_{3} \\
e_{1} & 0 & e_{3} & e_{2} \\
e_{2} & e_{3} & 0 & e_{1} \\
e_{3} & e_{2} & e_{1} & 0 \\
\hline
\end{array}
$$

Figure 1. Quaternion multiplication table.

relation tables and structure constants for the most popular in physical applications Lie algebras.

For the octonions ( $n=7$ ) we get octonions multiplication table (see Figure 2), where $e_{a}^{2}=-1 ; a=1,2, \cdots, 7$. From (1) it follows that $e_{a}$ are the traceless, antihermition generators of octonian algebra. Then the structure constants $f_{a b c}$ may be computed from

$$
e_{a} \times e_{b} \equiv \frac{1}{2}\left[e_{a}, e_{b}\right]=f_{a b c} e_{c} ; \quad a, b, c=1, \cdots, 7
$$

where $f_{a b c}=\varepsilon_{a b c}$, and $\varepsilon_{a b c}$ is the totally antisymmetric analog of the Levi-Civita symbol in seven-dimensional vector space with only nonzero independent components

$$
f_{a b c}=f_{123}=f_{246}=f_{435}=f_{651}=f_{572}=f_{714}=f_{367}=1
$$

\section{Internal (Local Gauge) Symmetries}

Our knowledge of the physical system is expressed in terms of conserved measurable quantities. The Noether theorem provides the connection between them and the symmetry transformations which leave the equations of motion invariant.

Now let us consider the symmetries that play a major role in the description of the fundamental interactions. These are rank-one electromagnetic $\mathrm{U}(1)$ and its extension, the Weinberg-Salam-Glashow electroweak model $\mathrm{SU}(2) \otimes \mathrm{U}(1)$. Further, we use the second-rank extension of these-SU(3) of QCD and its close relative, G(2) [9]. In all the cases we have dealt with, the continuous Lie groups and algebras associate the transformations in the inner space of the particle with quantities measurable by macroscopic devices according to Noether theorem [10]. That connection is established by the universal relation

$$
\left[T_{a}, T_{b}\right]=i f_{a b c} T_{c}
$$

where $T_{a}$ are traceless, hermitian matrices, which we call gauge charges and $f_{a b c}$ are the structure constants that uniquely determine the symmetry group.

\subsection{The Lie Algebra of the SU(2) Group}

The group parameters form a three-dimensional vector space. As its base we choose standard Pauli matrices:

$$
T_{1}=\frac{1}{2}\left(\begin{array}{cc}
0 & 1 \\
1 & 0
\end{array}\right) ; \quad T_{2}=\frac{1}{2}\left(\begin{array}{cc}
0 & -i \\
i & 0
\end{array}\right) ; \quad T_{3}=\frac{1}{2}\left(\begin{array}{cc}
1 & 0 \\
0 & -1
\end{array}\right) .
$$

Here and in the following we use the normalization:

$$
\operatorname{Tr}\left\{T_{a} T_{b}^{+}\right\}=\frac{1}{2} \delta_{a b} ; a, b=1,2,3
$$

We use the common normalization convention in order to allow the comparison of vector spaces formed by consecutive Lie algebras. Then, structure constants $f_{a b c}$ are computed from (7). It is convenient to present the results in the form of a multiplication table (see Figure 3).

The $f_{a b c}=\varepsilon_{a b c}$ obtained is a totally anti-symmetric Levi-Civita symbol in three-dimensional vector space with only independent nonzero components $f_{123}=1$. 


\begin{tabular}{|cccccccc|}
\hline$e_{a} / e_{b}$ & $e_{1}$ & $e_{2}$ & $e_{3}$ & $e_{4}$ & $e_{5}$ & $e_{6}$ & $e_{7}$ \\
$e_{1}$ & 0 & $e_{3}$ & $e_{2}$ & $e_{7}$ & $e_{6}$ & $e_{5}$ & $e_{4}$ \\
$e_{2}$ & $e_{3}$ & 0 & $e_{1}$ & $e_{6}$ & $e_{7}$ & $e_{4}$ & $e_{5}$ \\
$e_{13}$ & $e_{2}$ & $e_{1}$ & 0 & $e_{3}$ & $e_{4}$ & $e_{7}$ & $e_{6}$ \\
$e_{4}$ & $e_{7}$ & $e_{6}$ & $e_{3}$ & 0 & $e_{3}$ & $e_{2}$ & $e_{1}$ \\
$e_{5}$ & $e_{6}$ & $e_{7}$ & $e_{5}$ & $e_{3}$ & 0 & $e_{1}$ & $e_{2}$ \\
$e_{6}$ & $e_{5}$ & $e_{4}$ & $e_{7}$ & $e_{2}$ & $e_{1}$ & 0 & $e_{3}$ \\
$e_{7}$ & $e_{4}$ & $e_{5}$ & $e_{6}$ & $e_{1}$ & $e_{2}$ & $e_{3}$ & 0 \\
\hline
\end{tabular}

Figure 2. Octonions multiplication table.

$$
\begin{array}{|cccc|}
\hline T_{a} / T_{b} & T_{1} & T_{2} & T_{3} \\
T_{1} & 0 & T_{3} & T_{2} \\
T_{2} & T_{3} & 0 & T_{1} \\
T_{3} & T_{2} & T_{1} & 0 \\
\hline
\end{array}
$$

Figure 3. Lie algebra and structure constants of SU(2).

\subsection{The Lie Algebra of the SU(3) Group}

Here we have to deal with eight group parameters. In order to maintain the connection with the Lie algebra of the SU(2) group we choose traceless, hermitian Gell-Mann matrices as the base of our vector space:

$$
\begin{aligned}
& T_{1}=\frac{1}{2}\left(\begin{array}{ccc}
0 & 1 & 0 \\
1 & 0 & 0 \\
0 & 0 & 0
\end{array}\right) ; T_{2}=\frac{1}{2}\left(\begin{array}{ccc}
0 & -i & 0 \\
i & 0 & 0 \\
0 & 0 & 0
\end{array}\right) ; T_{3}=\frac{1}{2}\left(\begin{array}{ccc}
1 & 0 & 0 \\
0 & -1 & 0 \\
0 & 0 & 0
\end{array}\right) ; T_{4}=\frac{1}{2}\left(\begin{array}{ccc}
0 & 0 & 1 \\
0 & 0 & 0 \\
1 & 0 & 0
\end{array}\right) ; \\
& T_{5}=\frac{1}{2}\left(\begin{array}{ccc}
0 & 0 & -i \\
0 & 0 & 0 \\
i & 0 & 0
\end{array}\right) ; T_{6}=\frac{1}{2}\left(\begin{array}{ccc}
0 & 0 & 0 \\
0 & 0 & 1 \\
0 & 1 & 0
\end{array}\right) ; T_{7}=\frac{1}{2}\left(\begin{array}{ccc}
0 & 0 & 0 \\
0 & 0 & -i \\
0 & i & 0
\end{array}\right) ; T_{8}=\frac{1}{2 \sqrt{3}}\left(\begin{array}{ccc}
1 & 0 & 0 \\
0 & 1 & 0 \\
0 & 0 & -2
\end{array}\right) .
\end{aligned}
$$
4). where

$$
f_{123}=1 ; \quad f_{147}=f_{516}=f_{246}=f_{257}=f_{345}=f_{637}=\frac{1}{2} ; \quad f_{458}=f_{678}=\frac{\sqrt{3}}{2}
$$

are non-vanishing, totally anti-symmetric structure constants.

\subsection{The Lie Algebra of the G2 Group}

The general elements of the G2 Lie algebra are described by fourteen parameters. The standard base is given in terms of fourteen $7 \times 7$ traceless hermitian matrices [11]: 


\begin{tabular}{|ccccccccc|}
\hline$T_{a} / T_{b}$ & $T_{1}$ & $T_{2}$ & $T_{3}$ & $T_{4}$ & $T_{5}$ & $T_{6}$ & $T_{7}$ & $T_{8}$ \\
$T_{1}$ & 0 & $T_{3}$ & $T_{2}$ & $T_{7}$ & $T_{6}$ & $T_{5}$ & $T_{4}$ & 0 \\
$T_{2}$ & $T_{3}$ & 0 & $T_{1}$ & $T_{6}$ & $T_{7}$ & $T_{4}$ & $T_{5}$ & 0 \\
$T_{3}$ & $T_{2}$ & $T_{1}$ & 0 & $T_{5}$ & $T_{4}$ & $T_{7}$ & $T_{6}$ & 0 \\
$T_{4}$ & $T_{7}$ & $T_{6}$ & $T_{5}$ & 0 & $T_{3}$ & $T_{2}$ & $T_{1}$ & $T_{5}$ \\
$T_{5}$ & $T_{6}$ & $T_{7}$ & $T_{4}$ & $T_{3}$ & 0 & $T_{1}$ & $T_{2}$ & $T_{4}$ \\
$T_{6}$ & $T_{5}$ & $T_{4}$ & $T_{7}$ & $T_{2}$ & $T_{1}$ & 0 & $T_{3} / T_{8}$ & $T_{7}$ \\
$T_{7}$ & $T_{4}$ & $T_{5}$ & $T_{6}$ & $T_{1}$ & $T_{2}$ & $T_{3} / T_{8}$ & 0 & $T_{6}$ \\
$T_{8}$ & 0 & 0 & 0 & $T_{5}$ & $T_{4}$ & $T_{7}$ & $T_{6}$ & 0 \\
\hline
\end{tabular}

Figure 4. Lie algebra and structure constants of SU(3).

$$
\begin{aligned}
& T_{1}=\frac{1}{2 \sqrt{2}}\left(\begin{array}{ccccccc}
0 & 1 & 0 & 0 & 0 & 0 & 0 \\
1 & 0 & 0 & 0 & 0 & 0 & 0 \\
0 & 0 & 0 & 0 & 0 & 0 & 0 \\
0 & 0 & 0 & 0 & -1 & 0 & 0 \\
0 & 0 & 0 & -1 & 0 & 0 & 0 \\
0 & 0 & 0 & 0 & 0 & 0 & 0 \\
0 & 0 & 0 & 0 & 0 & 0 & 0
\end{array}\right), T_{2}=\frac{1}{2 \sqrt{2}}\left(\begin{array}{ccccccc}
0 & -i & 0 & 0 & 0 & 0 & 0 \\
i & 0 & 0 & 0 & 0 & 0 & 0 \\
0 & 0 & 0 & 0 & 0 & 0 & 0 \\
0 & 0 & 0 & 0 & -i & 0 & 0 \\
0 & 0 & 0 & i & 0 & 0 & 0 \\
0 & 0 & 0 & 0 & 0 & 0 & 0 \\
0 & 0 & 0 & 0 & 0 & 0 & 0
\end{array}\right) \\
& T_{3}=\frac{1}{2 \sqrt{2}}\left(\begin{array}{ccccccc}
1 & 0 & 0 & 0 & 0 & 0 & 0 \\
0 & -1 & 0 & 0 & 0 & 0 & 0 \\
0 & 0 & 0 & 0 & 0 & 0 & 0 \\
0 & 0 & 0 & -1 & 0 & 0 & 0 \\
0 & 0 & 0 & 0 & 1 & 0 & 0 \\
0 & 0 & 0 & 0 & 0 & 0 & 0 \\
0 & 0 & 0 & 0 & 0 & 0 & 0
\end{array}\right), T_{4}=\frac{1}{2 \sqrt{2}}\left(\begin{array}{ccccccc}
0 & 0 & 1 & 0 & 0 & 0 & 0 \\
0 & 0 & 0 & 0 & 0 & 0 & 0 \\
1 & 0 & 0 & 0 & 0 & 0 & 0 \\
0 & 0 & 0 & 0 & 0 & -1 & 0 \\
0 & 0 & 0 & 0 & 0 & 0 & 0 \\
0 & 0 & 0 & -1 & 0 & 0 & 0 \\
0 & 0 & 0 & 0 & 0 & 0 & 0
\end{array}\right) \\
& T_{5}=\frac{1}{2 \sqrt{2}}\left(\begin{array}{ccccccc}
0 & 0 & -i & 0 & 0 & 0 & 0 \\
0 & 0 & 0 & 0 & 0 & 0 & 0 \\
i & 0 & 0 & 0 & 0 & 0 & 0 \\
0 & 0 & 0 & 0 & 0 & -i & 0 \\
0 & 0 & 0 & 0 & 0 & 0 & 0 \\
0 & 0 & 0 & i & 0 & 0 & 0 \\
0 & 0 & 0 & 0 & 0 & 0 & 0
\end{array}\right), T_{6}=\frac{1}{2 \sqrt{2}}\left(\begin{array}{ccccccc}
0 & 0 & 0 & 0 & 0 & 0 & 0 \\
0 & 0 & 1 & 0 & 0 & 0 & 0 \\
0 & 1 & 0 & 0 & 0 & 0 & 0 \\
0 & 0 & 0 & 0 & 0 & 0 & 0 \\
0 & 0 & 0 & 0 & 0 & -1 & 0 \\
0 & 0 & 0 & 0 & -1 & 0 & 0 \\
0 & 0 & 0 & 0 & 0 & 0 & 0
\end{array}\right) \\
& T_{7}=\frac{1}{2 \sqrt{2}}\left(\begin{array}{ccccccc}
0 & 0 & 0 & 0 & 0 & 0 & 0 \\
0 & 0 & -i & 0 & 0 & 0 & 0 \\
0 & i & 0 & 0 & 0 & 0 & 0 \\
0 & 0 & 0 & 0 & 0 & 0 & 0 \\
0 & 0 & 0 & 0 & 0 & -i & 0 \\
0 & 0 & 0 & 0 & i & 0 & 0 \\
0 & 0 & 0 & 0 & 0 & 0 & 0
\end{array}\right), T_{8}=\frac{1}{2 \sqrt{6}}\left(\begin{array}{ccccccc}
1 & 0 & 0 & 0 & 0 & 0 & 0 \\
0 & 1 & 0 & 0 & 0 & 0 & 0 \\
0 & 0 & -2 & 0 & 0 & 0 & 0 \\
0 & 0 & 0 & -1 & 0 & 0 & 0 \\
0 & 0 & 0 & 0 & -1 & 0 & 0 \\
0 & 0 & 0 & 0 & 0 & 2 & 0 \\
0 & 0 & 0 & 0 & 0 & 0 & 0
\end{array}\right)
\end{aligned}
$$




$$
\begin{aligned}
& T_{9}=\frac{1}{2 \sqrt{6}}\left(\begin{array}{ccccccc}
0 & 0 & 0 & 0 & 0 & 0 & \sqrt{2} \\
0 & 0 & 0 & 0 & 0 & -1 & 0 \\
0 & 0 & 0 & 0 & 1 & 0 & 0 \\
0 & 0 & 0 & 0 & 0 & 0 & \sqrt{2} \\
0 & 0 & 1 & 0 & 0 & 0 & 0 \\
0 & -1 & 0 & 0 & 0 & 0 & 0 \\
\sqrt{2} & 0 & 0 & \sqrt{2} & 0 & 0 & 0
\end{array}\right), T_{10}=\frac{1}{2 \sqrt{6}}\left(\begin{array}{ccccccc}
0 & 0 & 0 & 0 & 0 & 0 & i \sqrt{2} \\
0 & 0 & 0 & 0 & 0 & i & 0 \\
0 & 0 & 0 & 0 & -i & 0 & 0 \\
0 & 0 & 0 & 0 & 0 & 0 & -i \sqrt{2} \\
0 & 0 & i & 0 & 0 & 0 & 0 \\
0 & -i & 0 & 0 & 0 & 0 & 0 \\
-i \sqrt{2} & 0 & 0 & i \sqrt{2} & 0 & 0 & 0
\end{array}\right) \\
& T_{11}=\frac{1}{2 \sqrt{6}}\left(\begin{array}{ccccccc}
0 & 0 & 0 & 0 & 0 & 1 & 0 \\
0 & 0 & 0 & 0 & 0 & 0 & \sqrt{2} \\
0 & 0 & 0 & -1 & 0 & 0 & 0 \\
0 & 0 & -1 & 0 & 0 & 0 & 0 \\
0 & 0 & 0 & 0 & 0 & 0 & \sqrt{2} \\
1 & 0 & 0 & 0 & 0 & 0 & 0 \\
0 & \sqrt{2} & 0 & 0 & \sqrt{2} & 0 & 0
\end{array}\right), T_{12}=\frac{1}{2 \sqrt{6}}\left(\begin{array}{ccccccc}
0 & 0 & 0 & 0 & 0 & -i & 0 \\
0 & 0 & 0 & 0 & 0 & 0 & i \sqrt{2} \\
0 & 0 & 0 & i & 0 & 0 & 0 \\
0 & 0 & -i & 0 & 0 & 0 & 0 \\
0 & 0 & 0 & 0 & 0 & 0 & -i \sqrt{2} \\
i & 0 & 0 & 0 & 0 & 0 & 0 \\
0 & -i \sqrt{2} & 0 & 0 & i \sqrt{2} & 0 & 0
\end{array}\right) \\
& T_{13}=\frac{1}{2 \sqrt{6}}\left(\begin{array}{ccccccc}
0 & 0 & 0 & 0 & -1 & 0 & 0 \\
0 & 0 & 0 & 1 & 0 & 0 & 0 \\
0 & 0 & 0 & 0 & 0 & 0 & \sqrt{2} \\
0 & 1 & 0 & 0 & 0 & 0 & 0 \\
-1 & 0 & 0 & 0 & 0 & 0 & 0 \\
0 & 0 & 0 & 0 & 0 & 0 & \sqrt{2} \\
0 & 0 & \sqrt{2} & 0 & 0 & \sqrt{2} & 0
\end{array}\right), T_{14}=\frac{1}{2 \sqrt{6}}\left(\begin{array}{ccccccc}
0 & 0 & 0 & 0 & i & 0 & 0 \\
0 & 0 & 0 & -i & 0 & 0 & 0 \\
0 & 0 & 0 & 0 & 0 & 0 & i \sqrt{2} \\
0 & i & 0 & 0 & 0 & 0 & 0 \\
-i & 0 & 0 & 0 & 0 & 0 & 0 \\
0 & 0 & 0 & 0 & 0 & 0 & -i \sqrt{2} \\
0 & 0 & -i \sqrt{2} & 0 & 0 & i \sqrt{2} & 0
\end{array}\right)
\end{aligned}
$$

And thus we obtain the corresponding multiplication table (see Figure 5).

\begin{tabular}{|ccccccccccccccc|}
\hline$T_{a} / T_{b}$ & $T_{1}$ & $T_{2}$ & $T_{3}$ & $T_{4}$ & $T_{5}$ & $T_{6}$ & $T_{7}$ & $T_{8}$ & $T_{9}$ & $T_{10}$ & $T_{11}$ & $T_{12}$ & $T_{13}$ & $T_{14}$ \\
$T_{1}$ & 0 & $T_{3}$ & $T_{2}$ & $T_{7}$ & $T_{6}$ & $T_{5}$ & $T_{4}$ & 0 & $T_{12}$ & $T_{11}$ & $T_{10}$ & $T_{9}$ & 0 & 0 \\
$T_{2}$ & $T_{3}$ & 0 & $T_{1}$ & $T_{6}$ & $T_{7}$ & $T_{4}$ & $T_{5}$ & 0 & $T_{11}$ & $T_{12}$ & $T_{9}$ & $T_{10}$ & 0 & 0 \\
$T_{3}$ & $T_{2}$ & $T_{1}$ & 0 & $T_{5}$ & $T_{4}$ & $T_{7}$ & $T_{6}$ & 0 & $T_{10}$ & $T_{9}$ & $T_{12}$ & $T_{11}$ & 0 & 0 \\
$T_{4}$ & $T_{7}$ & $T_{6}$ & $T_{5}$ & 0 & $T_{3} / T_{8}$ & $T_{2}$ & $T_{1}$ & $T_{5}$ & $T_{14}$ & $T_{13}$ & 0 & 0 & $T_{10}$ & $T_{9}$ \\
$T_{5}$ & $T_{6}$ & $T_{7}$ & $T_{4}$ & $T_{3} / T_{8}$ & 0 & $T_{1}$ & $T_{2}$ & $T_{4}$ & $T_{13}$ & $T_{14}$ & 0 & 0 & $T_{9}$ & $T_{10}$ \\
$T_{6}$ & $T_{5}$ & $T_{4}$ & $T_{7}$ & $T_{2}$ & $T_{1}$ & 0 & $T_{3} / T_{8}$ & $T_{7}$ & 0 & 0 & $T_{14}$ & $T_{13}$ & $T_{12}$ & $T_{11}$ \\
$T_{7}$ & $T_{4}$ & $T_{5}$ & $T_{6}$ & $T_{1}$ & $T_{2}$ & $T_{3} / T_{8}$ & 0 & $T_{6}$ & 0 & 0 & $T_{13}$ & $T_{14}$ & $T_{11}$ & $T_{12}$ \\
$T_{8}$ & 0 & 0 & 0 & $T_{5}$ & $T_{4}$ & $T_{7}$ & $T_{6}$ & 0 & $T_{10}$ & $T_{9}$ & $T_{12}$ & $T_{11}$ & $T_{14}$ & $T_{13}$ \\
$T_{9}$ & $T_{12}$ & $T_{11}$ & $T_{10}$ & $T_{14}$ & $T_{13}$ & 0 & 0 & $T_{10}$ & 0 & $T_{3} / T_{8}$ & $T_{2}$ & $T_{1}$ & $T_{5}$ & $T_{4}$ \\
$T_{10}$ & $T_{11}$ & $T_{12}$ & $T_{9}$ & $T_{13}$ & $T_{14}$ & 0 & 0 & $T_{9}$ & $T_{3} / T_{8}$ & 0 & $T_{1}$ & $T_{2}$ & $T_{4}$ & $T_{5}$ \\
$T_{11}$ & $T_{10}$ & $T_{9}$ & $T_{12}$ & 0 & 0 & $T_{14}$ & $T_{13}$ & $T_{12}$ & $T_{2}$ & $T_{1}$ & 0 & $T_{3} / T_{8}$ & $T_{7}$ & $T_{6}$ \\
$T_{12}$ & $T_{9}$ & $T_{10}$ & $T_{11}$ & 0 & 0 & $T_{13}$ & $T_{14}$ & $T_{11}$ & $T_{1}$ & $T_{2}$ & $T_{3} / T_{8}$ & 0 & $T_{6}$ & $T_{7}$ \\
$T_{13}$ & 0 & 0 & 0 & $T_{10}$ & $T_{9}$ & $T_{12}$ & $T_{11}$ & $T_{14}$ & $T_{5}$ & $T_{4}$ & $T_{7}$ & $T_{6}$ & 0 & $T_{8}$ \\
$T_{14}$ & 0 & 0 & 0 & $T_{9}$ & $T_{10}$ & $T_{11}$ & $T_{12}$ & $T_{13}$ & $T_{4}$ & $T_{5}$ & $T_{6}$ & $T_{7}$ & $T_{8}$ & 0 \\
\hline
\end{tabular}


where

$$
\begin{aligned}
& f_{123}=\frac{1}{\sqrt{2}} ; f_{147}=f_{516}=f_{246}=f_{257}=f_{345}=f_{637}=\frac{1}{2 \sqrt{2}} ; \\
& f_{1093}=f_{11123}=f_{1291}=f_{9414}=f_{11011}=f_{21012}=f_{5913}=f_{41013}=f_{51014}=f_{61411}=f_{71214}=f_{71113}=f_{61213}=\frac{1}{2 \sqrt{2}} ; \\
& f_{458}=f_{678}=\frac{\sqrt{3}}{2 \sqrt{2}} ; \quad f_{9810}=f_{81211}=\frac{1}{2 \sqrt{6}} ; \quad f_{81314}=\frac{1}{\sqrt{6}}
\end{aligned}
$$

are non-vanishing, totally anti-symmetric structure constants.

Notice that SU(2) Pauli matrices as well as SU(3) Gell-Mann matrices do not allow some general form that can describe all the matrices. I guess that the G2 (T1 to T14) also do not allow to do so.

\section{Equations of Motion of Non-Abelian Waves}

Consider an elementary particle whose motion is parametrized by the external position $x_{j} ; j=1,2,3$; velocity $\dot{x}_{j} ; j=1,2,3$; and internal gauge charges $T_{a} ; a=1, \cdots, n$, which are the non-Abelian analogs of electromagnetic charge; $n$ is the dimension of the vector space formed by those charges. Then the defining commutation relations are

$$
\begin{aligned}
& {\left[x_{j}, x_{k}\right]=0} \\
& {\left[x_{j}, \dot{x}_{k}\right]=i \delta_{j k}} \\
& {\left[T_{a}, T_{b}\right]=i f_{a b c} T_{c}} \\
& {\left[x_{j}, T_{a}\right]=0}
\end{aligned}
$$

where $j, k=1,2,3$ and $a, b, c=1, \cdots, n$.

In general, the equations of particle motion are Newtonian equations

$$
m \ddot{x}_{j}=F_{j}(x, \dot{x}, t)
$$

and generalized Wong's equations [2]

$$
\dot{T}_{a}+g f_{a b c} A_{j}^{b} T^{c} \dot{x}_{j}=0 ; j=1,2,3 ; a, b, c=1, \cdots, n
$$

(in the time axial gauge $A_{0}=0$ ). $A_{j}$ is the vector potentials of the external gauge fields

$$
A_{j}=A_{j}^{a} T_{a} ; j=1,2,3 ; a, b, c=1, \cdots, n
$$

Particle motion affected by the generalized Lorentz force

$$
F_{j}(x, \dot{x}, t)=g E_{j}(x, t)+g \varepsilon_{j k l} \dot{x}_{k} B_{l}(x, t)
$$

where

$$
E_{j}(x, t) \equiv E_{j}^{a}(x, t) T_{a} \text { and } B_{j}(x, t) \equiv B_{j}^{a}(x, t) T_{a}
$$

are three-dimensional vectors in outer particle space and $n$ - dimensional vectors in the inner particle space. They are the expected solutions of the generalized Yang-Mills [12]-Shaw [13]-Lee [3]-Wong [2] equations

$$
\begin{gathered}
\partial_{j} B_{j}^{a}+g f^{a b c} A_{j}^{b} B_{j}^{c}=0 \\
\frac{\partial B_{i}^{a}}{\partial t}+\varepsilon_{i j k}\left(\partial_{j} E_{k}^{a}+g f^{a b c} A_{j}^{b} E_{k}^{c}\right)=0 \\
\partial_{i} E_{i}^{a}+g f^{a b c} A_{i}^{b} E_{i}^{c}=\rho^{a} \\
-\frac{\partial E_{i}^{a}}{\partial t}+\varepsilon_{i j k}\left(\partial_{j} B_{k}^{a}+g f^{a b c} A_{j}^{b} B_{k}^{c}\right)=j_{i}^{a}
\end{gathered}
$$


In particular, for $n=1$ we have Maxwell-Faraday electromagnetic theory; for $n=3$ we have the WeinbergSalam-Glashow electroweak model; for $n=8$ we have SU(3) QCD and for $n=14$ we obtain G2 generalization of Yang-Mills theory that may also have some relevance to the unified theory of fundamental interactions.

However, neither SU(3) nor G2 are based on seven-dimensional space of internal parameters in obvious contradiction to the requirement that $n=7$ from [7] and [8].

\section{Color}

So far we have considered matrices with the real and complex matrix elements. There are numerous ways to obtain hypercomplex extensions of these matrices. The simplest is:

$$
\begin{aligned}
& e_{1}=-i \cdot\left(\begin{array}{cc}
0 & 1 \\
1 & 0
\end{array}\right)=-i \sigma_{1} ; \quad \sigma_{1}=i e_{1} \\
& e_{2}=-i \cdot\left(\begin{array}{cc}
0 & -i \\
i & 0
\end{array}\right)=-i \sigma_{2} ; \quad \sigma_{2}=i e_{2} \\
& e_{3}=-i \cdot\left(\begin{array}{cc}
1 & 0 \\
0 & -1
\end{array}\right)=-i \sigma_{3} ; \quad \sigma_{3}=i e_{3} .
\end{aligned}
$$

These expressions may be treated as a substitution of $2 \times 2$ matrices containing complex matrix elements by $1 \times 1$ matrices containing quaternion matrix elements. This treatment is legitimate since quaternions do allow for matrix representation while octonions, as stated previously, cannot be represented by matrices. Nevertheless, (25) give us an idea of how to introduce a special definition of charges and currents that do satisfy the $n=7$ requirement. Namely,

$$
T_{j}=i e_{j} ; j=1, \cdots, 7
$$

Then the structure constants are: $f_{a b c}=f_{123}=f_{246}=f_{435}=f_{651}=f_{572}=f_{714}=f_{367}=1$

We assume that these are the color charges and currents in the unified theory of the fundamental interactions. Our confidence is based on the discovery made by I. Newton [14]:

\begin{tabular}{ccccccc}
\hline Red & Orange & Yellow & Green & Blue & Indigo & Violet \\
\hline
\end{tabular}

\section{Conclusion}

It looks like a long way to go until the comparison with the experimental results could be obtained within this approach. However, definitely it provides an interesting extension of the current version of the quantum field theory.

\section{References}

[1] Dyson, F.J. (1990) American Journal of Physics, 58, 209-211. http://dx.doi.org/10.1119/1.16188

[2] Wong, S.K. (1970) Il Nuovo Cimento A, 65, 689-694. http://dx.doi.org/10.1007/BF02892134

[3] Lee, C.R. (1990) Physics Letters A, 148, 146-148. http://dx.doi.org/10.1016/0375-9601(90)90769-K

[4] Sepunaru, D. (2015) Journal of Modern Physics, 6, 698-709. http://dx.doi.org/10.4236/jmp.2015.65075

[5] Silagadze, Z.K. (2002) Annales de Fondation Louis de Broglie, 27, 241-255. http://arxiv.org/abs/hep-ph/0106235

[6] Tanimura, S. (1992) Annals of Physics, 220, 229-247. http://dx.doi.org/10.1016/0003-4916(92)90362-P

[7] Eckmann, B. (1943) Commentarii Mathematici Helvetici, 15, 318-339. http://dx.doi.org/10.1007/BF02565648

[8] Rost, M. (1996) Documenta Mathematica, 1, 209-214.

[9] Behrends, R.E., Dreitlein, J., Fronsdal, C. and Lee, W. (1962) Reviews of Modern Physics, 34, 1. http://dx.doi.org/10.1103/RevModPhys.34.1

[10] Noether, E. (1918) Gottingen Math. Physic., Nachr. kgl. Ges.. Wiss. Kl., 235-257.

[11] Carone, C.D. and Rastogi, A. (2008) Physical Review D, 77, 035011. http://dx.doi.org/10.1103/PhysRevD.77.035011

[12] Yang, C.N. and Mills, R.L. (1954) Physical Review, 96, 191- 201. http://dx.doi.org/10.1103/PhysRev.96.191 
[13] Shaw, R. (1955) Ph.D. Diss., Cambridge University, Cambridge, pt. II, ch. III.

[14] Newton, I. (1704) Opticks: Or, A Treatise of the Reflections, Refractions, Inflexions and Colours of Light. Also Two Treatises of the Species and Magnitude of Curvilinear Figures. London. 